\title{
Basic Photonic Wire Components in Silicon-on-insulator
}

\author{
Pieter Dumon*, Gunther Roelkens*, Wim Bogaerts*, Dries Van Thourhout*, \\ Johan Wouters ${ }^{\ddagger}$, Stephan Beckx ${ }^{\ddagger}$, Patrick Jaenen ${ }^{\ddagger}$ and Roel Baets* \\ * Photonics Research Group, Dept. of Information Technology, Ghent University - IMEC, Belgium \\ Pieter.Dumon@intec.UGent.be \\ $\ddagger$ IMEC, Silicon Process Technology Division, Leuven - Belgium
}

\begin{abstract}
We fabricated basic structures for photonic integrated circuits in Silicon-on-insulator photonic wires using CMOS technology. We show low-loss bends and waveguide intersections, power splitters, MMI's and Mach-Zehnder interferometers.
\end{abstract}

\section{INTRODUCTION}

Photonic wire waveguides in silicon-on-insulator (SOI) confine light within submicron dimensions by total internal reflection. Ultra-compact, low-loss bends promise high density photonic integrated circuits. Passive SOI photonic wire technology can be very well suited for functions such as wavelength routing filters. In this context, we are investigating basic building blocks for SOI photonic wire-based circuits, fabricated using CMOS technology. In order to build complex circuits, these basic building blocks, from simple bends to more complex interferometric structures, must be well known. It is important to complement simulations with experiments to get a realistic idea about what performance can be achieved and what complexity levels will be possible in future circuits. Loss phenomena are important, but also at interfaces, considerable refraction- and diffraction effects occur which are very dependent on the actually fabricated geometry.

Structures were created in $200 \mathrm{~mm}$ SOI wafers with a Silicon layer thickness of $220 \mathrm{~nm}$ and a $1 \mu \mathrm{m}$ burried oxide. A deep UV lithography stepper with a $248 \mathrm{~nm}$ illumination wavelength defines patterns in resist. A dry etching process is used to transfer the patterns into the Silicon layer. A detailed overview of the processing steps can be found in [1]. The processes are basically CMOS processes, characterized and adapted for the fabrication of photonic circuits, which enforces quite different boundary conditions.

We characterized the structures using a tuneable laser and detector, using grating couplers for coupling to standard single-mode fibre [2]. These couplers have a $-7.5 \mathrm{~dB}$ maximum transmission and a $60 \mathrm{~nm} 3 \mathrm{~dB}$ bandwidth. Measured spectra shown hereafter are normalized on the transmission spectrum of a short wire (including fibre couplers).

\section{WIRES AND BENDS}

In order to measure straight and bend losses accurately, we fabricated spiral-like structures with different total lengths and different number of $90^{\circ}$ bends. With a good choice of

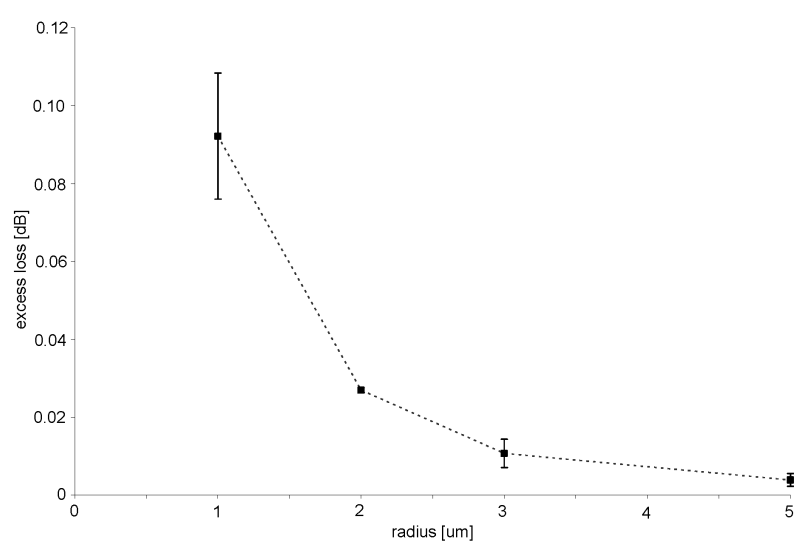

Fig. 1. Excess bend loss of a 90 degree bend connected to two straight waveguides, as a function of radius. The standard deviation of the fit is indicated.

the samples in this parameter space, a reasonable fit of the propagation losses in straight wires and excess bend losses can be made. For 1,2 and $3 \mu \mathrm{m}$ radius, structures with a total length up to $25 \mathrm{~mm}$ and up to $55090^{\circ}$ bends have been fabricated. For $5 \mu \mathrm{m}$ radius, total length varied up to $50 \mathrm{~mm}$. The fitted excess bend loss includes the mode mismatch at the interfaces between straight and bent waveguides. Another possible source of bend loss is coupling to the TM mode which is very lossy. However, this effect is difficult to quantify both experimentally and by simulation.

Measurements show that for $5 \mu \mathrm{m}$ radius, losses are completely dominated by the normal propagation losses. For $1 \mu \mathrm{m}$ radius on the other hand, excess bend losses dominate. Results for a 540nm wide wire are plotted in Figure 1. Excess bend losses for $5 \mu \mathrm{m}$ radius are smaller than $0.004 \mathrm{~dB}$. Propagation losses of straight wires are about $0.26 \mathrm{~dB} / \mathrm{mm}$ in this case.

Additionally, structures were fabricated with many $90^{\circ}$ bends and small offsets $(0-25 \mathrm{~nm})$ between straight and bent wire sections. For $5 \mu \mathrm{m}$ radius, the offset for which optimal tranmission is achieved is neglible at $540 \mathrm{~nm}$ and around $5 \mathrm{~nm}$ for $480 \mathrm{~nm}$ width. For a $3 \mu \mathrm{m}$ radius, the optimal offset is 15 $20 \mathrm{~nm}$. 


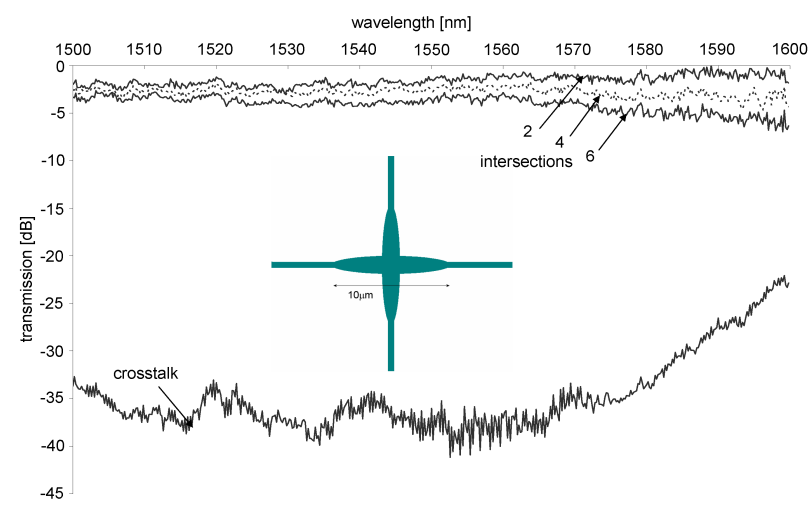

Fig. 2. Normalized transmission spectra of 2,4, and 6 consecutive tapered intersection and crosstalk. Losses are lower than $0.65 \mathrm{~dB} /$ intersection at $1540 \mathrm{~nm}$.

\section{LOW-LOSS WAVEGUIDE INTERSECTIONS}

In highly integrated circuits, connections between multiple components will inevitably cross. With only one level of wiring, waveguides will physically intersect each other, giving rise to losses and crosstalk. As this occurs already when combining only a few components at relatively low complexity levels and broadband ultra-low-loss intersections are not easy to achieve, waveguide crossings might even prove to be one of the main bottlenecks towards complex circuits.

The standard intersection without tapering theoretically experiences $1.4 \mathrm{~dB}$ insertion loss and a quite high crosstalk of about $-9 \mathrm{~dB}$ [3]. In [3], elliptically tapered intersections were studied and four-fold symmetric intersections fabricated with e-beam lithography showed losses of $1.2 \mathrm{~dB}$. The insertion loss measured for a standard intersection was almost $2 \mathrm{~dB}$.

We fabricated structures with 2, 4 and 6 four-fold symmetric elliptically tapered intersections. Losses lower than $0.65 \mathrm{~dB}$ per crossing are achieved. The crosstalk to the perpendicular waveguide in the first intersection was measured to be lower than $30 \mathrm{~dB}$ over a broad wavelength range. This is illustrated in Figure 2. While these are close to the simulated values, it is clear that still only a few intersections can be supported with acceptable loss.

\section{POWER SPLITTERS AND MMI'S}

We fabricated Y-splitters and 1x2 multimode interferometric (MMI) devices as balanced splitters. The MMI splitters used tapered waveguides in order to reduce insertion losses and relax fabrication tolerances. Simulation showed a 3x7.6 $\mu \mathrm{m}$ MMI with input- and outputwaveguides tapered to $1 \mu \mathrm{m}$ width has an insertion loss smaller than $0.1 \mathrm{~dB}$. First measured devices show $<1 \mathrm{~dB}$ insertion loss and very good balance between both outputs. Moreover, the MMI's allow for broadband operation.

Several Y-splitter types were designed, with both tapered and untapered input and output waveguides. Insertion losses are still around $1.5 \mathrm{~dB}$ however. Further optimization is needed both in the theoretical design and the mask design, in order to optimize the narrow gap between the output waveguides,

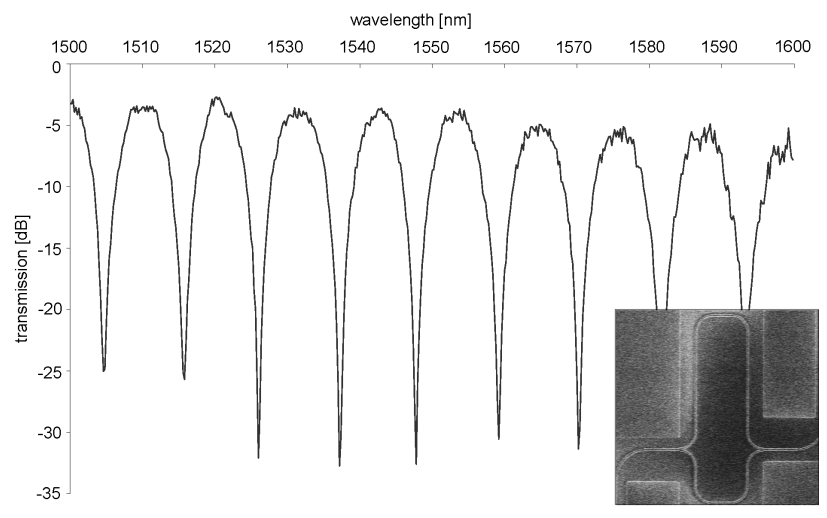

Fig. 3. Normalized transmission of a $1 \mathrm{x} 1 \mathrm{MZI}$ with $50 \mu \mathrm{m}$ delay length.

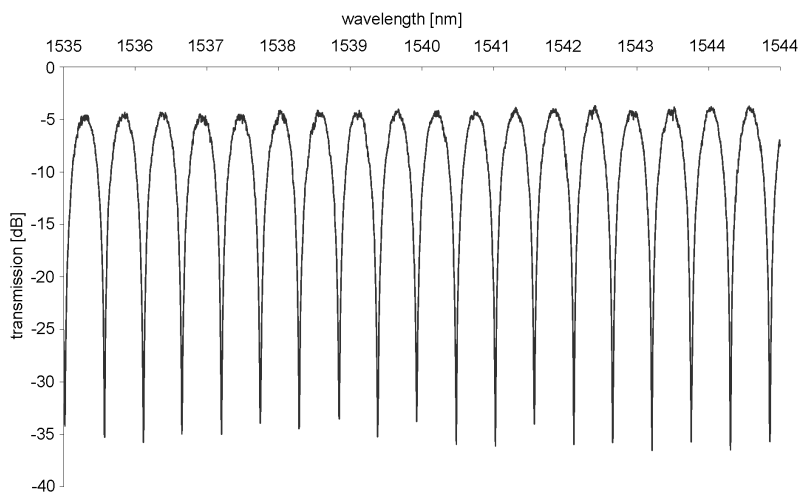

Fig. 4. Normalized transmission of a $1 \mathrm{x} 1 \mathrm{MZI}$ with $1 \mathrm{~mm}$ delay length.

which is quite critical. Also, we see a slight unbalance which can be due to small fabrication variations or mask digitization error.

\section{MACH-ZEHNDER INTERFEROMETERS}

One building block for integrated circuits is the MachZehnder interferometer (MZI). We fabricated 1x1 MZI's with delay lengths from $20 \mu \mathrm{m}$ up to $1 \mathrm{~mm}$ in one arm, corresponding to $27 \mathrm{~nm}$ down to $550 \mathrm{pm}$ free spectral range(FSR), respectively. The compact Y-splitters used were not well optimized yet, therefore still showing quite some excess loss.

Figures 3 and 4 show the transmission spectra (including fibre couplers) of MZI structures with $50 \mu \mathrm{m}$ and $1 \mathrm{~mm}$ delay lengths respectively. The insertion loss of around $4 \mathrm{~dB}$ is mostly due to Y-splitter loss. An extinction ratio larger than $25 \mathrm{~dB}$ is achieved. From the extinction ratio, insertion loss and known wire propagation losses, the splitter unbalance was estimated to be smaller than $0.13 \mathrm{~dB}$ around $1540 \mathrm{~nm}$ wavelength. For $50 \mu \mathrm{m}$ delay length, the FSR is around $11 \mathrm{~nm}$. Comparing MZI structures with different delay lengths allows us to accurately measure modal effective and group indices.

\section{All-PASS RING RESONATORS}

Earlier, we demonstrated ring resonators coupled to two waveguides, acting as add-drop filters [4]. Here, illustrate the 


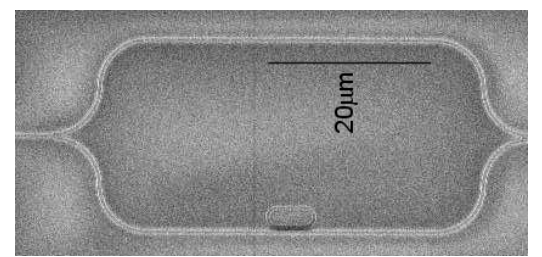

Fig. 5. SEM photo of a $1 \times 1$ Mach-Zehnder with an all-pass ring resonator

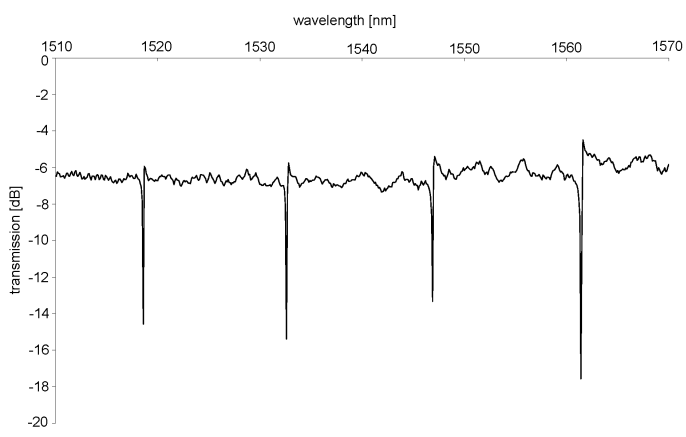

Fig. 6. Normalized transmission of a 1x1 MZI with a racetrack resonator in one arm. Radius of the resonator is $5 \mu \mathrm{m}$.

use of ring resonators as all-pass filters. An all-pass filter is embedded into an otherwise symmetric 1x1 Mach-Zehnder interferometer (MZI). The resonator is acting as a delay line with a non-linear phase response. Off resonance, the phase response of a lossless all-pass resonator is flat and a $2 \pi$ multiple. Around resonance, There is a fast $2 \pi$ phase jump, causing the MZI output to go through destructive interference. With a lossy resonator, the situation is a little different but basically the at-resonance extinction ratio in the MZI output is decreasing with increasing loss. From the maximum transmission, extinction ratio and extinction bandwidth, the ring losses and the coupling between ring and waveguide can be extracted. Figure 6 shows the measured transmission spectrum of such a device, composed of MZI and a racetrack resonator with $5 \mu \mathrm{m}$ radius and relatively low coupling between the racetrack and the waveguide. The $3 \mathrm{~dB}$ bandwidth around destructive interference is about $0.1 \mathrm{~nm}$ and the extinction ratio is $5-9 \mathrm{~dB}$. Due to optical proximity effects during lithography, the wire is narrower in the coupling region. This introduces an extra phase shift in the arm with the resonator, causing asymmetry in the spectrum around resonance. This can be seen in Fig. 6. Due to the unoptimized lithography mask design, this phase shift is a considerable fraction of $\pi$. The insertion loss is again dominated by the loss in the Y-splitters. The fitted power coupling strength between resonator and waveguide is just above $4 \%$. The losses in the resonator amount to $2 \mathrm{~dB} / \mathrm{mm}$ when averaged over the ring. However, we think most of this originates from the coupling section.

\section{CONCLUSION}

We fabricated basic Silicon-on-insulator photonic wire structures using CMOS processing techniques. Simple $90^{\circ}$ bends bends experience low loss of $<0.004 \mathrm{~dB}$ for $5 \mu \mathrm{m}$ radius. These losses were measured using structures with up to 550 bends and $50 \mathrm{~mm}$ total wire length. Tapered intersections show loss and crosstalk close to the calculated values. However, intersections might still be one of the bottlenecks towards real integration. MMI-based power splitters with tapered input and output waveguides have low excess loss and very good balance. 1x1 Mach-Zehnder interferometers show more than $25 \mathrm{~dB}$ extinction at destructive interference in the output.

\section{ACKNOWLEDGMENT}

The authors would like to thank Jimmy Mentens, Joost Van Ongeval and the other CMOS pilot line people for definition of the processing jobs, help and fabrication.

This work was supported by the EU through the ISTPICMOS project and the IST-ePIXnet network of excellence supported by the European Union. Part in this work has been performed in the context of the Belgian IAP PHOTON Network.

P. Dumon thanks the Institute for the Promotion of Innovation through Science and Technology in Flanders (IWTVlaanderen) for a scholarship. G. Roelkens and W. Bogaerts acknowledge the Flemish Fund for Scientific Research (FWOVlaanderen) for financial support.

\section{REFERENCES}

[1] W. Bogaerts et al., "Nanophotonic waveguides in silicon-on-insulator fabricated with cmos technology," J. Lightwave Technol., vol. 23, no. 1, pp. 401-412, January 2005.

[2] D. Taillaert et al., "An out-of-plane grating coupler for efficient buttcoupling between compact planar waveguides and single-mode fibers," IEEE J. Quantum Electron., vol. 38, no. 7, pp. 949-955, 2002.

[3] T. Fukazawa, T. Hirano, F. Ohno, and T. Baba, "Low loss intersection of si photonic wire waveguides," Jap. J. of Applied Physics, vol. 43, no. 2, pp. 646-647, 2004.

[4] P. Dumon et al., "Microring resonators in silicon-on-insulator," in ECIO 2005, Grenoble, 4 2005, pp. 196-199. 